\title{
BMJ Open How do general practitioners and pharmacists experience antibiotic use in out-of-hours primary care? An exploratory qualitative interview study to inform a participatory action research project
}

\author{
Annelies Colliers, ${ }^{1}$ Samuel Coenen, ${ }^{1,2,3}$ Roy Remmen, ${ }^{1}$ Hilde Philips, ${ }^{1}$ \\ Sibyl Anthierens ${ }^{1}$
}

To cite: Colliers A, Coenen S, Remmen $\mathrm{R}$, et al. How do general practitioners and pharmacists experience antibiotic use in out-of-hours primary care? An exploratory qualitative interview study to inform a participatory action research project. BMJ Open 2018;8:e023154. doi:10.1136/ bmjopen-2018-023154

- Prepublication history and additional material for this paper are available online. To view these files, please visit the journal online (http://dx.doi. org/10.1136/bmjopen-2018023154).

$\mathrm{AC}$ and $\mathrm{SC}$ contributed equally.

Received 23 March 2018

Revised 2 July 2018

Accepted 29 August 2018

D) Check for updates

(c) Author(s) (or their employer(s)) 2018. Re-use permitted under CC BY-NC. No commercial re-use. See rights and permissions. Published by BMJ.

For numbered affiliations see end of article.

Correspondence to Dr. Sibyl Anthierens; Sibyl.Anthierens@uantwerpen. be

\section{ABSTRACT}

Rationale Antibiotics (ABs) are one of the most prescribed medications in out-of-hours $(\mathrm{OOH})$ care in Belgium. Developing a better understanding of why ABs are prescribed in this setting is essential to improve prescribing habits.

Objectives To assess $\mathrm{AB}$ prescribing and dispensing challenges for general practitioners (GPS) and pharmacists in $\mathrm{OOH}$ primary care, and to identify context-specific elements that can help the implementation of behaviour change interventions to improve $\mathrm{AB}$ prescribing in this setting. Design This is an exploratory qualitative study using semistructured interviews. This study is part of a participatory action research project.

Setting and participants Participants include 17 GPs and 1 manager, who work in a Belgian $00 \mathrm{H}$ general practitioners cooperative (GPC), and 5 pharmacists of the area covered by the GPC. The GPC serves a population of more than 187000 people.

Results GPs feel the threshold to prescribe $A B$ in $00 \mathrm{H}$ care is lower in comparion to office hours. GPs and pharmacists talk about the difference in their professional identity in $\mathrm{OOH}$ (they define their task differently, they feel more isolated, insecure, have the need to please and so on), type of patients (unknown patients, vulnerable patients, other ethnicities, demanding patients and so on), workload (they feel time-pressured) and lack of diagnostic tools or follow-up. They are aware of the problem of $A B$ overprescribing, but they do not feel ownership of the problem.

Conclusion The implementation of behaviour change interventions to improve $\mathrm{AB}$ prescribing in $00 \mathrm{H}$ primary care has to take these context specifics into account and could involve interprofessional collaboration between GPs and pharmacists.

Trial registration number NCT03082521; Pre-results.

\section{INTRODUCTION}

Antibiotic resistance is a growing problem caused by unnecessary and suboptimal
Strengths and limitations of this study

- Participants talked very openly and specifically about the challenges of antibiotic use in out-of-hours $(\mathrm{OOH})$ primary care; however, participants might not be representative of the whole $00 \mathrm{H}$ setting.

- Findings in this study can help to develop tailored and bottom-up supported solutions to improve antibiotic prescribing in $\mathrm{OOH}$ primary care.

This study confirms the results of previous research on this topic and adds relevant context-specific elements.

- The study gained a wide range of opinions by including different stakeholders of $\mathrm{OOH}$ primary care.

- These findings may be transferable to different primary healthcare systems depending on the context (patients, healthcare workers, organisation of $\mathrm{OOH}$ care, payment and reimbursement systems, prescribing habits, and so on).

quality of antibiotic prescribing. The highest proportion of antibiotics is being prescribed in primary care. Despite all the efforts throughout the last two decades, Belgium stays one of the highest prescribing countries in Europe. ${ }^{1}$

The establishment of general practitioners cooperatives (GPCs) to cover out-of-hours $(\mathrm{OOH})$ care represents one of the most important developments for primary healthcare in Flanders, Belgium. ${ }^{2-4}$ A GPC offers an innovative way to address many general practitioners (GPs), collaborating at one site, and unprecedented opportunities to improve the quality of their work in $\mathrm{OOH}$, but also with possible spillover effects during office hours. Antibiotics are one of the most prescribed medications in $\mathrm{OOH}$ care in Belgium. ${ }^{5-7}$ 
The interest in antibiotic prescribing in $\mathrm{OOH}$ care and possible corresponding interventions is growing internationally. ${ }^{8-13}$ The results of earlier intervention studies show us a rather limited positive effect on antibiotic prescribing in size, quality and time. These observations evoke the hypothesis that top-down intervention might have only small results. In addition the implementation of these interventions and the realisation of an effective change in real practice remain a big societal challenge. Continuing this reasoning, we will study the effects of bottom-up developed interventions on antibiotic prescribing.

This study is part of the BAbAR (Better Antibiotic prescribing through Action Research) project, which uses the participatory action research (PAR) approach, to improve antibiotic prescribing of GPs in an OOH GPC. ${ }^{14}$ In the first exploratory phase of the BAbAR project, we develop a partnership with the GPC and map barriers and opportunities to implement change interventions. This manuscript is part of this particular phase to describe and understand the context. In a second phase we will focus on facilitating change and implementing interventions through the Plan-Do-Study-Act cycles. In a third phase antibiotic prescribing quality outside and antibiotic use during office hours will be evaluated. Equally important are the process evaluation and theory building on improving antibiotic prescribing. PAR is an appropriate method to develop a tailored quality improvement intervention in dialogue with the relevant stakeholders. ${ }^{15} 16$ This approach systematically analyses and accounts for the many contextual, cultural and behavioural factors involved in local antimicrobial prescribing, to optimise intervention effectiveness. Although the evidence in this field is still limited, PAR is promising to optimise antibiotic prescribing behaviour, and solutions are needed now more than ever. ${ }^{17-19}$ The literature on different types of interventions to improve antibiotic prescribing is growing as is the interest in why interventions work or not. ${ }^{20-23}$ When working on interventions to improve antibiotic prescribing, studying and reflecting on the specifics of the context and setting are important to come up with tailored bottom-up supported solutions. ${ }^{822} 2425$ To optimise the quality of GPs' antibiotic prescribing behaviour in $\mathrm{OOH}$ care, a better understanding of the present attitudes as well as interventions that build on this knowledge is needed. Prescribing behaviour is about making a diagnosis and implementing a treatment plan, and about social interactions in a specific context. To develop an understanding of the specifics of antibiotic use in $\mathrm{OOH}$ care and the possible solutions for inappropriate prescribing, interviews were done with GPs, GPC management and pharmacists of a GPC region. Pharmacists were interviewed because of their first-line interaction with patients having infections (over-the-counter medication, self-care advice, health-seeking advice and so on) and experience with dispensing antibiotics. Working as a GP or pharmacist in $\mathrm{OOH}$ primary care is not the same as working during office hours. Therefore, this study sets out to map the specific challenges of prescribing or dispensing antibiotics when dealing with patients with infections in $\mathrm{OOH}$ primary care.

\section{METHODS}

We set up a qualitative study to gain insight into the experiences of stakeholders of the GPC with antibiotic prescribing and dispensing in $\mathrm{OOH}$ care, the problems and possible solutions. The purpose was to develop a better understanding of why antibiotics are prescribed in this setting.

\section{Study design}

Face-to-face interviews were conducted by AC. GPs know her as a former colleague. This gives her a unique insider-outsider role. ${ }^{26}$ To be able to stand outside the situation and reflect on it, but also to know the context as an insider and to feel part of the group, can help the research as well as the action forward. In a few interviews GPs talked about the role of the pharmacist on call, which made us decide to also interview pharmacists to explore certain responses from the interviews with the GPs.

The interviews followed a semistructured interview guide (online supplementary file 1). We reflected on our interview questions by reviewing the previous work, exploring GP and nurse prescriber's views on and experiences of prescribing antibiotics for respiratory tract infections in primary care $\mathrm{OOH}$ services in the $\mathrm{UK}^{8}{ }^{8} \mathrm{We}$ refined the questions through a dialogic and iterative process after the first two interviews.

The interviews were audio-taped and transcribed verbatim afterwards.

Each interview was divided into three parts. The first part is discussed in this article and included questions on the why and when antibiotics are prescribed in $\mathrm{OOH}$ care, how it might differ from office hours, local antibiotic prescribing culture and habits, opportunities and need for change, ideas on prescribing profile and feedback, and so on. In the second part, the idea of PAR was explained and questions were asked on the ideas on and involvement with PAR. The third part focused on GPs' views about using video recordings of their consultations in $\mathrm{OOH}$ care as a research and learning tool.

\section{Study setting and population}

Belgian GPs have no formal gatekeeper function. There is free access to primary, secondary and tertiary care. Belgian OOH care is being organised more often in GPCs since the last decade. Every GP is obliged to participate in this rotation-based system of being on call during the weekends in his own region. ${ }^{4}$

The GPC of the Antwerp city centre covers more than 187000 inhabitants. One hundred and seventy GPs (92 women, 21 GP trainees) work in shifts of 12 hours in this GPC during the weekend. Their average age is 49.3 years. The weekday nights are covered by the same group of GPs but not from the GPC location, but from their own practices. In the weekend 3 out of 85 pharmacies in the region 
will be open during daytime. The night shift is covered by two pharmacies.

To ensure anonymity of the interviewees, their quotes will only be labelled with the age group $(25+$ years, $30+$ years, $35+$ years...) and professional group (G for GP or GPC manager, and $\mathrm{P}$ for pharmacist).

GPs working in the GPC were purposively sampled to reflect the variety of these GPs' own practices. They were approached by email or telephone. The planned sample size before the study started was 15-20 GPs. We stopped interviewing when data saturation was reached and when we had a sufficient reflection of the heterogeneous group of GPs. Additionally we purposively sampled five pharmacists, based on location, age and gender.

\section{Patient and public involvement}

In this stage of the BAbAR project, patients were not yet involved. In the second 'facilitating change' phase of the study, the perspective of patients will be included in assessing the feasibility and acceptability of the implemented interventions to improve antibiotic use.

\section{Analysis}

All interview transcripts were systematically analysed according to the thematic analysis method. ${ }^{27} 28$

The first step was to read and familiarise with the data and to start critically appraising what the data mean. The first three interviews were coded by two researchers independently: AC (GP, PhD student) and SA (postdoctoral medical sociologist). In a second step, initial codes were generated that appeared meaningful by doing complete coding. ${ }^{29}$ After the initial coding the two researchers (AC, SA) independently examined the codes and started to create potential patterns and merged them into themes. After this independent coding stage, the two researchers convened to discuss the initial thematic framework. Similarities and differences were discussed and amended to create a set of themes that represent both analyses. The interim analyses were critically assessed by the other members of the research team and adapted after their feedback. The thematic framework was used for further analysis and adapted as new ideas appeared in the data. All interviews were checked again with the new thematic framework (constant comparison). Themes were refined and defined in a last step. Data coding and analysis was supported by using Quirkos. ${ }^{30}$

Scientific rigour was ensured by using researchers' triangulation and member checks involving the participants of the interviews in order to ensure that the findings make sense to the participants and to explore whether they wanted to add other elements to the findings. For this reason the GPs, GPC manager and pharmacists who have contributed to the interviews received a formal analysis report with the summary of the findings and were asked to provide feedback.
Table 1 Characteristics of the interviewees

\begin{tabular}{|c|c|}
\hline \multicolumn{2}{|l|}{ Participants (n) } \\
\hline General practitioners (GP) & 17 \\
\hline Pharmacists & 5 \\
\hline General practitioners cooperative manager & 1 \\
\hline Mean (SD) age (years) - GPs & $48.2(12.75)$ \\
\hline Mean (SD) age (years)-pharmacists & $41.8(11.95)$ \\
\hline Mean (SD) number of years in practice & $22.1(12.51)$ \\
\hline Mean (SD) number of years in pharmacy & $16.8(12.38)$ \\
\hline \multicolumn{2}{|l|}{ Gender distribution } \\
\hline Male & 8 \\
\hline Female & 15 \\
\hline \multicolumn{2}{|l|}{$\begin{array}{l}\text { Type of GP practice during inhours } \\
\text { care }(n=17)\end{array}$} \\
\hline Solo & 4 \\
\hline Duo & 4 \\
\hline Group & 8 \\
\hline Community health centre & 1 \\
\hline $\begin{array}{l}\text { GP trainee in the GP practice inhours care } \\
(n=17)\end{array}$ & 8 \\
\hline Mean duration of the interviews $(\mathrm{min})^{*}-\mathrm{GPs}$ & 48 \\
\hline $\begin{array}{l}\text { Mean duration of the interviews }(\min )^{\star}- \\
\text { pharmacists }\end{array}$ & 32 \\
\hline
\end{tabular}

${ }^{*}$ Parts 1, 2 and 3, with part 1 on prescribing antibiotics in out-ofhours care, part 2 on participatory action research and part 3 on video recordings of consultations.

\section{RESULTS}

Twenty GPs and the GPC manager were invited to participate; three refused to participate. A good range of the different districts of Antwerp, gender, years of experience and type of practice was obtained. Five pharmacists were invited and agreed to participate. Twenty-three interviews were conducted (table 1).

Additional quotes can be found in online supplementary file 2.

The data show that the participants hold a different threshold to prescribe antibiotics during $\mathrm{OOH}$ than during office hours. They describe some reasons why the setting influences their prescribing behaviour as they experience a different professional identity in $\mathrm{OOH}$ care, the profile of the patient is different and the setting as a whole is more stressful. Participants do acknowledge that there is a problem with overprescribing of antibiotics, but they do not take ownership of the problem. These dimensions are discussed in detail below.

\section{Theme 1: the threshold to prescribe antibiotics differs} between $\mathrm{OOH}$ and during office hours, the choice of antibiotic does not

The GPs talk about the threshold to prescribe an antibiotic at the GPC and compare this with their office hours practice. Most GPs say their threshold to prescribe antibiotics is lower in $\mathrm{OOH}$ care. Some GPs say they more often 
use a wait-and-see policy. Others say there is no difference. However, all GPs claim that there is no difference in the choice of antibiotics.

The pharmacists notice that some GPs prefer one specific product, often broad-spectrum antibiotics or quinolones, so they deliver more of that product during a weekend shift. Sometimes that even means they run out of stock of that specific antibiotic when the choice of antibiotic is unusual.

Sometimes it happens ... that I see the same kind of prescription a dozen of times during one shift ... and then you know: that doctor is in the mood for that one product that day. And then I run out of stock if they choose those more uncommon antibiotics. (P2, female, 37 years)

\section{Theme 2: reasons (not) to prescribe antibiotics in $\mathbf{0 O H}$ care}

The GPs sum up numerous reasons why their antibiotic prescribing habits in $\mathrm{OOH}$ care differ from that during office hours. Some of these reasons explain why they are more prone to write antibiotics in $\mathrm{OOH}$ care; others demonstrate the opposite.

\section{Professional identity in a GPC differs from working in their own} practice

\section{Working at the GPC}

Working at the GPC is experienced differently as working in their own practice.

First of all they talk about working at the GPC as working in a 'vacuum'. The GPs do not feel the same connection with the GPC as with their own practice. They have worked hard during the week and coming to the GPC feels as an extra burden. They feel less accountable for the decisions they make and are less restrictive because of a lack of energy.

Let it be clear that you are different as a GP on call than a GP in your own practice. In my practice I have a kind of guerrilla attitude, like 'I'm not going to give antibiotics, whatever the patient says, I stick to my scientific guideline'. That guerrilla attitude I have far less at the GPC. ... Like, well, I followed the rules already for 5 days during the week, and then I want to put up a fight, than I can be a guerrilla: no, you can't get antibiotics. (G4, female, $50+$ years)

For some of them the interpretation of their role of being the GP on call is identifying and treating the urgent and serious cases, a triage function, and afterwards referring patients to their regular GP. So for them it is easier to refuse antibiotics during $\mathrm{OOH}$ care or to tell patients to wait and see their own GP when problems continue or worsen.

\section{Safety and insecurity}

Safety and insecurity issues were discussed a lot, often related to patient and $\mathrm{OOH}$ specifics, such as not knowing the patient, different mother tongue, time pressure and so on. The GPs are afraid to miss serious bacterial infections or to be confronted with complications when not treating.

There are always patient factors that give some pressure. On the one hand you feel more free, because you say, well it's not my patient and if they're not happy they have to see for themselves and then they could go see their own doctor. But on the other hand you feel less secure and you go play on the safe side. The pressure to make mistakes is bigger at the GPC. (G6, male, $60+$ years)

And then there is the loyalty and responsibility the GP in $\mathrm{OOH}$ feels towards the patient's own GP, for example, when patients explain that with this problem they always receive antibiotics from their regular GP.

\section{Reciprocity and pleasing}

Belgian healthcare is mostly organised on a 'fee for service' basis, and thus the patient pays for the consultation directly after seeing the GP. The GPC can choose for a third-party payment, but it is only used occasionally and is meant for deprived patients. Patients have obligatory medical insurance, which will reimburse the costs partly. However, the GPs feel a sense of reciprocity when seeing patients. This quid-pro-quo principle makes it difficult to make patients pay for a consultation where 'nothing' substantial, such as an antibiotic prescription, is given in return.

I think that plays a role ... you can't send them home and say: 'well just continue what you have been doing for the last three weeks'. And then to ask 60 euro or something? Unconsciously that plays a role. Like it costs them money to come here, it has to be worth it. ... I have to be an added value, because they did an effort to come over... (G4, female, $50+$ years)

The GPs also feel they need to please patients. They want to resolve the patient's problems and offer (quick) solutions. This makes it difficult to bring the message that the infection will pass, without intervening, and that the patient just has to wait.

Also the pharmacists talk about wanting to relieve the symptoms quickly in order to help the patient, and they try to do this with over-the counter medication. A commercial element also plays a role in their work.

We give some acetylcysteine when there is mucus, or some coughing syrup with an upper airway infection, or some cranberry or mannitol for a urinary tract infection, but if it is a bacterial infection, it won't work ... We are not doctors. But you want to help them. ( $\mathrm{P} 5$, male, 49 years)

Dextromethorphan syrup is dextromethorphan syrup, no matter which brand it is, so yes a good profit margin is important then. ( $\mathrm{P} 4$, female, 38 years)

Some GPs talk about the effort it takes to bring the message that the infection is self-limiting and to convince 
the patient that it will pass without the use of antibiotics. Just giving a prescription for antibiotics is easier and quicker. They communicate differently at the GPC. Others feel that it is their job to educate the patient, certainly at the GPC.

\section{Hide behind common standards}

Several interviewees suggested to make a specific GPC guideline on antibiotic prescribing. GPs like the fact that they can hide behind a decision that was made not by themselves, but for example by the GPC board. They referred to the agreement at the GPC not to prescribe benzodiazepines to patients, because the need for this type of medication can only be evaluated by their own GP, which helps GPs on call to refuse this type of prescription. It is not me that decides this, 'we as a GPC' don't do this type of medicine. And if every GP stops prescribing (antibiotics), patient pressure will go down. The GPC board supports this idea.

But of course, it's a utopia, if no one would prescribe it, then it will come naturally again to say no ... which I find very positive about the prescriptions for sleeping pills for example. I feel so comfortable to say: This is no longer prescribed at the GPC, ... they sometimes say, 'I always get antibiotics', and then I think yes, if all GPs would stop prescribing antibiotics, a policy, where generally less is prescribed, that would help me (G8, female, $40+$ years)

\section{Patient profile}

\section{The 'unknown' patient}

The 'unknown' patient is the most frequently discussed item. You do not know the medical background of patients, and it is difficult to assess how the patient normally expresses his symptoms. There is no shared electronic health record for the GP on call.

The thing that makes it difficult at the GPC, is the fact that it are all new faces, people I don't know. In my own practice I do not take any new patients, so all I have here is people I know well. I already know who complains about what, and whether I should take that complaint seriously or not. (G13, male, $60+$ years)

The pharmacists have a similar experience during the weekend. They see more 'unknown' people during the weekend. Or often a relative visits the pharmacy, and they only have an incomplete history to work with.

\section{'Other type' of patients}

Often patients visiting the GPC have already consulted their own GP during the week, but the symptoms are not yet resolved. Then it is difficult to repeat the same message of wait-and-see and the GPs feel extra pressure to prescribe antibiotics.

Patients seen at the GPC sometimes differ from those the GPs see at their day-to-day practice. Therefore, they do not always feel confident in treating them. For example a lot of small sick children visit the GPC. Not every GP feels comfortable with this age group. This is the same for the elderly at home or in a nursing home. For those vulnerable populations the threshold to prescribe antibiotics is lower because the GPs may fear bad prognosis.

The GPC and the pharmacist on call attract a lot of patients from different ethnicities, who do not always speak a language they understand and who have different cultural views on how to treat infections. Educating these patients takes time and energy they do not always have when they are on call.

Patients seeking help during the weekend at the GPC, according to some, are a specific kind of patients. The GPs feel that they are less prone to rely on self-care, for example. They are more doctor-oriented and intervention-minded, focused on getting a simple solution.

Maybe the population that visits the GPC is more like: 'and now it has to be resolved'. That it is a type of people that expects that. Besides you have a bunch of people who never visit a GPC, unless there is a real calamity, so ... I think there are more of the "consuming type' at the GPC. (G8, female, $40+$ years)

The GPs and pharmacists talked about the limited general and personal medical knowledge patients have, for example, on the natural evolution and the self-limiting characteristic of infectious diseases and on having a real penicillin allergy or not. This, combined with the lack of access to a personal medical health record, makes them feel uncertain.

\section{The GPC, a stressful environment without diagnostic tools or follow-up}

The access to diagnostic tools at the GPC is still limited. There is no radiology at the site. It is logistically difficult to analyse blood samples. There are no point-of-care tests available, except for a urine stick or human chorionic gonadotropin (HCG) test. This means organising these technical tests for a patient takes time and effort (contacting a neighbouring hospital, contacting the lab and so on).

It's more difficult than a day at your own practice, because you have far less diagnostic possibilities. At my own practice I can say, I draw blood, I will send you for an X-ray, and so I do things to get it clearer if it is viral or bacterial. At the GPC that's more difficult. So you are more in doubt and you can't do anything. Or you can send them to the hospital, that's the only way to get some clarity. But I don't think the emergency departments will be happy with that. So in situations where you don't want to take any risks, like the elderly or young children, you do give antibiotics. (G7, female, $45+$ years)

Although $\mathrm{C}$ reactive protein point-of-care tests are not yet common practice in Belgium, many GPs know they exist, have read about this and suggest to implement this at the GPC. 
The GP on call often feels time pressure, for example, by a full waiting room or a colleague working faster. Sometimes it is the pressure put on them by the patient, for example, because they have a flight planned or they have to work.

The fact that they cannot follow up the patient themselves is sometimes also a reason to prescribe quicker at the GPC.

I know you can wait quite a bit according to the guideline before you give antibiotics for sinusitis, but I see patients at the GPC that are really in pain, and then I think: 'Do I have to let them wait for another 5 days?' That's more difficult than in your own practice.

I: why is that more difficult?

Eumh well, maybe it's a bit the time pressure as well. And also the fact that you are working with two doctors, and you can see how fast the other one is going. Like: Oh no, he is seeing his third patient, and I'm still seeing the first. (G10, female, $60+$ years)

Also the pharmacists talk about time pressure resulting in less communication.

It's much busier than during the normal hours ... So it's more executing, you are less critical, because you have less time, ..., if they come with a prescription, I just deliver. I explain the usage, but I won't ask questions anymore, the diagnosis was already made. (P2, female, 37 years)

\section{Theme 3: antibiotic overprescribing: aware but not owner of the problem \\ Awareness of the problem}

Most GPs are familiar with the local guidelines on infectious disease management and antibiotic prescribing. There is a difference in how they deal with these guidelines. Some use them actively and will check them regularly while working with patients. Others claim they know what is in there, and they do not need to check anymore. Some stick to their own antibiotic preferences, although they know their choice is not guideline-recommended. They have experienced problems with antimicrobial resistance in their own patients, resulting in a more prudent use in some GPs and to fear the use of broad-spectrum antibiotics in others.

The GPs and pharmacists all state that they are aware of the problem of antimicrobial resistance and the importance of restrictive antibiotic use. They all refer positively to the public awareness campaigns and the evolution in the public opinion on antibiotics in the last decades.

\section{No ownership of the problem}

The GPs had a vague idea of their prescribing profile, compared with colleagues. Many referred to their colleagues' antibiotic prescribing habits. In most cases it was labelled as irrational, not guideline-recommended, too much and too quickly, playing safe, the easy way, and so on. The younger GPs and pharmacists said it was mostly a problem among the older GPs. Some of the older GPs said that the younger ones were more insecure and prescribed more.

No, I do not prescribe antibiotics lightly, but I sometimes see something else, and maybe that's more with the younger colleagues, I don't know if there is a trend, that they prescribe antibiotics too quickly at the GPC, and that's not the place to do that, that's my vision. (G15, male, $55+$ years)

Mostly the older doctors prescribe lightly. The younger ones are stricter. (P1, female, 58 years)

\section{Feedback and the electronic medical health record}

GPs suggested providing personal feedback on their antibiotic prescribing allowing comparisons with their colleagues.

GPs receive a medical report on each of their own patients seen by the GP on call during the weekend. They refer to this report as inadequate (no diagnosis, little information on clinical examination and so on). Often they cannot assess if it was really necessary to prescribe an antibiotic or not. Also the assumption was made that some diagnoses were exaggerated in the report to justify antibiotic prescribing. And there are a few older GPs who will not work with a computer.

\section{DISCUSSION}

We learnt from our participants that prudent antibiotic prescribing is even more difficult in the $\mathrm{OOH}$ setting than during office hours, as reported in the literature on prudent antibiotic prescribing during office hours. ${ }^{31}$ The context of work is different during office hours, which makes it more difficult to prescribe prudently, and GPs experience their professional identity at the GPC differently, which has an influence on their antibiotic prescribing decisions. Encounters with unknown patients, vulnerable groups such as elderly and children, or foreign-language patients make GPs play on the safe side, that is, they prescribe more easily. Furthermore, the GPC setting lacks diagnostic tools, patient information or follow-up.

We could confirm some of the specific $\mathrm{OOH}$ care elements which are also discussed in the UNderstanding medIcal and non-medical anTibiotic prescribing for rEspiratory tract infections in primary care out of hours services (UNITE) study, such as lack of follow-up of patients, limited access to patient medical records, vulnerable patients, time pressure and feeling of having to do something. ${ }^{8}$ But some factors are locally defined and were different: Belgium healthcare does not work with nurse prescribers, participation in the $\mathrm{OOH}$ system is mandatory for every GP, there is no triage, lack of diagnostic tools, no engagement to working in the GPC (working in a vacuum), not feeling accountable (no ownership of the problem) and so on, leading to even more uncertainty in treating patients than during office hours. ${ }^{31}$ Tailoring 
and implementing interventions in $\mathrm{OOH}$ care should be directed to these specific elements, and the process should be monitored to learn what works and what not. By exploring different questions-such as what are we doing exactly, how big is the problem, what could we do differently, and so on-we could create ownership of the problem, engagement to the problem and understanding. We will feed back the results of the interviews together with the prescribing feedback to the GPs and think together with them about possible solutions to improve prescribing, which is the aim of the second phase of the BAbAR study.

On the other hand GPs talk about elements that are playing a role in antibiotic prescribing that are very similar during office hours, as we can find in other studies. ${ }^{32}$ The need for reciprocity in a fee-for-service system is well known, diagnosis shifting to justify an antibiotic prescription, as well as the lack of ownership of the problem. ${ }^{32}$

Although pharmacists and GPs during $\mathrm{OOH}$ care have the same goal of 'caring for the patient' and recognise the importance of antimicrobial resistance, there is not much interprofessional collaboration yet on this topic in our setting. A more active role for the pharmacist could be considered when developing interventions. ${ }^{33-35}$ This has been successful in the Netherlands in reducing the number of antibiotic prescriptions for respiratory tract infections for adolescents and adults during office hours. ${ }^{36}$ Both are able and willing to take up their professional role in this topic. To do so we will have to take into account the interprofessional dynamics between pharmacists and GPs. ${ }^{37}$

\section{Strengths and limitations}

Antibiotic prescribing is an important issue in a high-prescribing country like Belgium. These interviews provide valuable insights into GPs' perceptions on the specific challenges of prudent antibiotic use in an $\mathrm{OOH}$ setting, and some issues have been discussed with pharmacists but need further indepth exploration. Some findings are related to and have to be seen within the context of Belgian $\mathrm{OOH}$ care, but are nonetheless transferable to other settings.

Participant sampling was purposeful and stakeholders were eager to cooperate. AC invited GPs and pharmacists to participate in the study and conducted the interviews. Many of the participants know her as a former colleague. This could have led to reticence in answering some of the questions or it could have enhanced the 'trusting environment'. We feel it has had a positive effect on the interviews as we collected a lot of self-critical and honest answers from the participants, and negative views were freely reported. All our participants received a summary of our findings and they had time to give comments on them. This can be seen as an extra step in the analysis process to enhance the trustworthiness of the results. All our participants were happy to receive the summary and had no additional comments.

\section{CONCLUSION}

Antibiotic use in primary $\mathrm{OOH}$ care has its own specifics due to GP, pharmacist, patient and setting-related issues. For a successful implementation of behaviour change interventions in $\mathrm{OOH}$ primary care, these multiple and interacting specifics should be taken into account. Involving pharmacists could have an added value.

\section{Author affiliations}

${ }^{1}$ Department of General Practice - Primary and Interdisciplinary Care, Faculty of Medicine and Health Sciences, University of Antwerp, Wilrijk, Belgium

${ }^{2}$ Vaccine and Infectious Disease Institute (VAXINFECTIO), Faculty of Medicine and Health Sciences, University of Antwerp, Wilrijk, Belgium

${ }^{3}$ Department of Epidemiology and Social Medicine (ESOC), Faculty of Medicine and Health Sciences, University of Antwerp, Wilrijk, Belgium

Acknowledgements We would like to thank the participating GPs, manager and pharmacists for their time and effort, and KAVA (Koninklijke Apothekersvereniging van Antwerpen), the Antwerp association of pharmacists.

Contributors AC, SC, RR, HP and SA contributed to the concept and design of the study, drafted and revised the manuscript, have given final approval of the version to be published and agree to be accountable for all aspects of the work.

Funding The study is part of the BAbAR study and has been granted a PhD fellowship by the Faculty of Medicine and Health Sciences of the University of Antwerp.

Competing interests None declared.

Patient consent Not required.

Ethics approval The study was approved by the Ethics Committee of Antwerp University Hospital/University of Antwerp (reference number 17/08/089).

Provenance and peer review Not commissioned; externally peer reviewed.

Data sharing statement Participants were invited to review the analysis before transferring copyright to the University of Antwerp for use in PAR research, teaching and publications. All authors had access to all of the data in the study and take responsibility for the integrity of the data and the accuracy of the data analysis. No additional data are available.

Open access This is an open access article distributed in accordance with the Creative Commons Attribution Non Commercial (CC BY-NC 4.0) license, which permits others to distribute, remix, adapt, build upon this work non-commercially, and license their derivative works on different terms, provided the original work is properly cited, appropriate credit is given, any changes made indicated, and the use is non-commercial. See: http://creativecommons.org/licenses/by-nc/4.0/.

\section{REFERENCES}

1. European Centre for Disease Prevention and Control, 2017. Summary of the latest data on antibiotic consumption in the European Union https://ecdc.europa.eu/en/publications-data/ summary-latest-data-antibiotic-consumption-eu-2017 (accessed 19 Jan 2018).

2. Huibers L, Philips H, Giesen P, et al. EurOOHnet-the European research network for out-of-hours primary health care. Eur J Gen Pract 2014;20:229-32.

3. Philips H, Mahr D, Remmen R, et al. Predicting the place of out-ofhours care--a market simulation based on discrete choice analysis. Health Policy 2012;106:284-90.

4. Philips H. Out-of-hours care in Belgium. Dissertation for the degree of doctor in Medical Science at the University of Antwerp, 2010:ISBN: 9789057283109.

5. Adriaenssens N, Bartholomeeusen S, Ryckebosch P, et al. Quality of antibiotic prescription during office hours and out-of-hours in Flemish primary care, using European quality indicators. Eur J Gen Pract 2014;20:114-20.

6. Bartholomeeusen S, Philips H, Van Royen P, et al. iCAREdata: improving care and research electronic data trust Antwerp: Zenodo, 2017.

7. Colliers A, Bartholomeeusen S, Remmen R, et al. Improving Care And Research Electronic Data Trust Antwerp (iCAREdata): a research 
database of linked data on out-of-hours primary care. BMC Res Notes 2016;9:259.

8. Williams SJ, Halls AV, Tonkin-Crine S, et al. General practitioner and nurse prescriber experiences of prescribing antibiotics for respiratory tract infections in UK primary care out-of-hours services (the UNITE study). J Antimicrob Chemother 2018;73:795-803.

9. Lindberg BH, Gjelstad S, Foshaug M, et al. Antibiotic prescribing for acute respiratory tract infections in Norwegian primary care out-ofhours service. Scand J Prim Health Care 2017;35:178-85.

10. Hayward GN, Fisher RF, Spence GT, et al. Increase in antibiotic prescriptions in out-of-hours primary care in contrast to in-hours primary care prescriptions: service evaluation in a population of 600000 patients. J Antimicrob Chemother 2016;71:2612-9.

11. Dyrkorn R, Gjelstad S, Espnes KA, et al. Peer academic detailing on use of antibiotics in acute respiratory tract infections. A controlled study in an urban Norwegian out-of-hours service. Scand J Prim Health Care 2016;34:180-5.

12. de Bont EG, Dinant GJ, Elshout G, et al. An illness-focused interactive booklet to optimise management and medication for childhood fever and infections in out-of-hours primary care: study protocol for a cluster randomised trial. Trials 2016;17:547.

13. Willems L, Denckens $\mathrm{P}$, Philips $\mathrm{H}$, et al. Can we improve adherence to guidelines for the treatment of lower urinary tract infection? A simple, multifaceted intervention in out-of-hours services. $J$ Antimicrob Chemother 2012;67:2997-3000.

14. Colliers A, Coenen S, Philips $\mathrm{H}$, et al. Optimising the quality of antibiotic prescribing in out-of-hours primary care in Belgium: a study protocol for an action research project. BMJ Open 2017;7:e017522.

15. Meyer J. Qualitative research in health care. Using qualitative methods in health related action research. BMJ 2000;320:178-81.

16. Meyer J. Evaluating action research. Age Ageing 2000;29(Suppl 2):8-10.

17. Sikkens JJ, van Agtmael MA, Peters EJG, et al. Behavioral approach to appropriate antimicrobial prescribing in hospitals: the Dutch Unique Method for Antimicrobial Stewardship (DUMAS) participatory intervention study. JAMA Intern Med 2017;177:1130-8.

18. van Buul LW, Sikkens JJ, van Agtmael MA, et al. Participatory action research in antimicrobial stewardship: a novel approach to improving antimicrobial prescribing in hospitals and long-term care facilities. $J$ Antimicrob Chemother 2014;69:1734-41.

19. Klepser ME, Dobson EL, Pogue JM, et al. A call to action for outpatient antibiotic stewardship. J Am Pharm Assoc 2017;57:457-63.

20. Coxeter P, Del Mar CB, McGregor L, et al. Interventions to facilitate shared decision making to address antibiotic use for acute respiratory infections in primary care. Cochrane Database Syst Rev 2015:CD010907.

21. Cross EL, Tolfree R, Kipping R. Systematic review of public-targeted communication interventions to improve antibiotic use. J Antimicrob Chemother 2017; 72:975-87.

22. Tonkin-Crine SK, Tan PS, van Hecke O, et al. Clinician-targeted interventions to influence antibiotic prescribing behaviour for acute respiratory infections in primary care: an overview of systematic reviews. Cochrane Database Syst Rev 2017;9:Cd012252.

23. van der Velden AW, Pijpers EJ, Kuyvenhoven MM, et al. Effectiveness of physician-targeted interventions to improve antibiotic use for respiratory tract infections. $\mathrm{Br} \mathrm{J}$ Gen Pract 2012;62:e801-7.

24. Papoutsi C, Mattick K, Pearson M, et al. Social and professional influences on antimicrobial prescribing for doctors-in-training: a realist review. J Antimicrob Chemother 2017;72:2418-30.

25. Strandberg EL, Brorsson A, Hagstam C, et al. "I'm Dr Jekyll and Mr Hyde": are GPs' antibiotic prescribing patterns contextually dependent? A qualitative focus group study. Scand J Prim Health Care 2013;31:158-65.

26. McNiff J, Whitehead J. All you need to know about action research California: SAGE, 2011.

27. Braun V, Clarke V. Using thematic analysis in psychology. Qual Res Psychol 2006;3:77-101.

28. Ritchie J, Spencer L. Qualitative data analysis for applied policy research. Analysing qualitative data. London: Routledge, 2002;573:305-29.

29. Braun V, Clarke V. Successful qualitative research: a practical guide for beginners. London: Sage, 2013.

30. Quirkos. Qualitative data analysis software. https://www.quirkos.com (accessed 19 Jan 2018)

31. Tonkin-Crine S, Yardley L, Coenen S, et al. Strategies to promote prudent antibiotic use: exploring the views of professionals who develop and implement guidelines and interventions. Fam Pract 2013;30:88-95.

32. Brookes-Howell L, Hood K, Cooper L, et al. Understanding variation in primary medical care: a nine-country qualitative study of clinicians' accounts of the non-clinical factors that shape antibiotic prescribing decisions for lower respiratory tract infection. BMJ Open 2012;2:e000796.

33. Roque F, Teixeira-Rodrigues A, Breitenfeld L, et al. Decreasing antibiotic use through a joint intervention targeting physicians and pharmacists. Future Microbiol 2016;11:877-86.

34. Roque F, Soares S, Breitenfeld L, et al. Attitudes of community pharmacists to antibiotic dispensing and microbial resistance: a qualitative study in Portugal. Int J Clin Pharm 2013;35:417-24.

35. Dey RM, de Vries MJ, Bosnic-Anticevich S. Collaboration in chronic care: unpacking the relationship of pharmacists and general medical practitioners in primary care. Int $J$ Pharm Pract 2011;19:21-9.

36. Vervloet M, Meulepas MA, Cals JW, et al. Reducing antibiotic prescriptions for respiratory tract infections in family practice: results of a cluster randomized controlled trial evaluating a multifaceted peer-group-based intervention. NPJ Prim Care Respir Med 2016;26:15083.

37. Broom A, Broom J, Kirby E, et al. The path of least resistance? Jurisdictions, responsibility and professional asymmetries in pharmacists' accounts of antibiotic decisions in hospitals. Soc Sci Med 2015;146:95-103. 